The Astrophysical Journal Supplement Series, 90:883-888, 1994 February

c) 1994. The American Astronomical Society. All rights reserved. Printed in U.S.A.

\title{
THE STATUS OF ULTRA-HIGH-ENERGY GAMMA-RAY OBSERVATIONS
}

\author{
R. J. Protheroe \\ Department of Physics and Mathematical Physics, University of Adelaide, S.A. 5000, Australia \\ Received 1993 March 1; accepted 1993 May 21
}

\begin{abstract}
In 1983 Samorski and Stamm found evidence of ultra-high-energy (UHE) gamma-ray emission from Cygnus X-3 in archival data from the Kiel air shower array. The emission appeared to be emitted only at one phase in the binary orbit. The Kiel array was sensitive to air showers generated by particles with energies above $3 \times 10^{15} \mathrm{eV}$. Their discovery led to searches in archival data from other arrays for evidence of UHE emission from Cygnus X-3 and other potential sources in the Galaxy, and to the construction of new air shower arrays dedicated to UHE astronomy.

In the mid 1980s many groups around the world claimed detections of Cygnus X-3, Hercules X-1, Vela X-1, and other objects. Cygnus X-3 is strongly variable at all wavelengths, and so it would be surprising if this variability did not extend to UHE gamma-rays also. This indeed appeared to be the case, and in the mid to late 1980s the UHE gamma-ray output showed a steady decline apart from transient UHE emission following giant radio flares. With the exception of possible detections above $10^{17} \mathrm{eV}$ by the Fly's Eye and Akeno Array, I am unaware of any statistically compelling claimed detections of this source reported over the past 4 years. The new arrays-CASA-MIA and SPASE - have not detected any of the claimed sources, and it seems likely that at least some of these early claims may have been premature. However, although none of the observations taken alone was of extremely high statistical significance, the observations of Cygnus X-3 by different groups showed a consistency which I feel cannot be ascribed to chance alone. The observations of Cygnus X-3 and the Crab Nebula/Pulsar are reviewed with particular reference to the results published during the last 5 years.
\end{abstract}

Subject headings: gamma rays: observations — pulsars: individual (Crab Nebula ) stars: individual (Cygnus X-3)

\section{INTRODUCTION}

Gamma ray emission at high energies must result from interactions of high-energy particles with matter, radiation, or magnetic fields. The highest densities of cosmic rays are likely to occur close to their acceleration sites, and so ultra-high-energy $\gamma$-ray observations give the potential to search for sites of particle acceleration in astrophysical environments. For $\gamma$-rays above $\sim 100 \mathrm{TeV}$, usually referred to as ultra-high-energy (UHE), interactions with the microwave background radiation limit how far we can observe sources (see, e.g., Protheroe 1986 and references therein). At $1 \mathrm{PeV}\left(10^{15} \mathrm{eV}\right)$ the mean interaction length is less than $10 \mathrm{kpc}$, effectively limiting UHE $\gamma$-ray astronomy to our Galaxy and the Magellanic Clouds. In the Galaxy and most astrophysical environments it is easier to accelerate protons and nuclei to UHE than electrons because of the higher synchrotron and other energy losses experienced by electrons. We know that cosmic rays with energies up to $10^{20} \mathrm{eV}$ do exist, and so the Galaxy must emit UHE $\gamma$-rays as a result of cosmic-ray interactions in the interstellar medium. The intensity of this diffuse emission is expected to be several orders of magnitude lower at $100 \mathrm{TeV}$ than that of the cosmic rays which form the background to UHE $\gamma$-ray observations. Upper limits to the diffuse $\gamma$-ray intensity from the Galactic plane from CASA MIA can already rule out a cosmic ray spectrum in the outer part of the Galaxy significantly flatter than that observed at Earth (Gaisser, Stanev, \& Halzen 1991).

Of greater interest is the possibility that some of the cosmic rays at UHE are accelerated at discrete sources in our Galaxy.
Interactions of some fraction of the accelerated cosmic rays in the source would give rise to potentially observable fluxes of UHE $\gamma$-rays which would then act as tracers of particle acceleration. Thus, UHE $\gamma$-ray observations have the potential to distinguish between a Galactic point source origin, diffuse Galactic origin (e.g., Ip \& Axford 1992) and an extragalactic origin (Protheroe \& Szabo 1992) of UHE cosmic rays.

At the highest energies, it becomes impractical to detect $\gamma$ rays directly with spacecraft or balloon experiments because of the low fluxes involved. Instead, one uses Earth's atmosphere as an essential part of a $\gamma$-ray telescope and detects at ground level the debris resulting from interactions of $\gamma$-rays with an air nucleus. Following an initial pair production, and subsequent bremsstrahlung interactions, a cascade rapidly develops and eventually dies away as the typical particle energies drop such that ionization losses and Compton scattering dominate over bremsstrahlung and pair production. The average altitude of maximum development depends on the energy of the primary $\gamma$-ray. At $1 \mathrm{TeV}$ the showers maximize typically at $\sim 10 \mathrm{~km}$ with $\sim 10^{3}$ electrons and have essentially died away before reaching sea level. At $1 \mathrm{PeV}$ the corresponding numbers are 6 $\mathrm{km}$ and $10^{6}$ electrons at maximum and $10^{5}$ at sea level. In the latter case, shower particles spread out over a few $100 \mathrm{~m}$ and they may be readily detected by an array of particle detectors which typically consist of $\sim 1 \mathrm{~m}^{2}$ of plastic scintillator viewed by a photomultiplier. A snapshot of the shower would reveal it to be a disk of particles of thickness a few meters, somewhat curved up at the edges, and traveling at speed $c$. Thus, by timing the arrival of shower particles in each array detector to 
an accuracy of a few nanoseconds one can reconstruct the arrival direction of the $\gamma$-ray. From the number of electrons detected one can estimate the total number of electrons at ground level, the "shower size," and get a crude measure of the $\gamma$-ray's energy.

At $\mathrm{TeV}$ energies, since too few shower particles reach ground level to be useful, one detects instead Cherenkov light emitted in the atmosphere by the shower electrons. For vertical showers, the light pool typically extends out to $150 \mathrm{~m}$ from the shower axis and the area of this light pool becomes the effective area of the optical Cherenkov telescope which consists typically of parabolic mirrors of area in excess of $10 \mathrm{~m}^{2}$, with one or more photomultipliers at their foci.

Unfortunately, cosmic-ray protons and nuclei also produce air showers, and it is impossible on a shower by shower basis to distinguish these from $\gamma$-ray-initiated showers. At TeV energies, one can reject a fraction of cosmic-ray showers on the basis of the shapes and orientation of their Cherenkov light images. These are more ragged than those of $\gamma$-ray showers which are also aligned such that they point toward the center of the field of view. At PeV energies, $\gamma$-ray showers have a lower muon content than cosmic-ray showers, and this may also be used to reject a fraction of the background events. In both cases the background rejection is far from complete. At PeV energies one uses the fact that the cosmic rays are essentially isotropic and arrive randomly in time to look for peaks consistent with the array's angular resolution (typically $\sim 1^{\circ}$ ) in the distribution of shower arrival directions and for periodicities in the arrival times consistent with known periods of the source.

In this review, I will concentrate on the UHE energy range accessible to air shower arrays. I will give a more optimistic, and therefore perhaps somewhat heretical, view about the UHE observations than is usually given (see, e.g., Weekes 1991 \& Ong 1992 for more pessimistic accounts). I will start with Cygnus X-3 in $\S 2$, and include a discussion of burst-type activity, EeV observations, and evidence for a $12.6 \mathrm{~ms}$ period. In $\S 3$ I will describe recent observations of the Crab Nebula and argue that this source is also variable at UHE. Space limitations prevent me from mentioning all claimed detections or upper limits of Cygnus X-3 and the Crab Nebula/Pulsar or from discussing observations of other sources at UHE. For comprehensive summaries of earlier observations, see the reviews by Protheroe (1987), Weekes (1988), Nagle, Gaisser, \& Protheroe (1988), Fegan (1990), and Rao \& Sreekantan (1992).

\section{CYGNUS $X-3$}

\subsection{Long-Term Observations}

The first UHE observations of Cygnus X-3 were made with the Kiel array which was originally designed for cosmic-ray studies. Samorski \& Stamm (1983) examined data taken between 1976 March and 1980 January and found a $4.4 \sigma$ DC excess from a bin with $\Delta \alpha=4^{\circ}$ and $\Delta \delta=3^{\circ}$ centered on Cygnus X-3. The events were found to be modulated with the $4.8 \mathrm{hr}$ binary period, the $\gamma$-ray emission occurring at phase $\phi \simeq$ 0.25 . The claimed flux above $2 \mathrm{PeV}$ was $7.4 \times 10^{-14} \mathrm{~cm}^{-2} \mathrm{~s}^{-1}$, and this corresponded to a luminosity of $6 \times 10^{36} \mathrm{ergs} \mathrm{s}^{-1}$ for energies in the range 2-20 PeV assuming isotropic emission by the source. If the $\gamma$-rays were produced as a result of accelera- tion of protons and interactions in the source, then the power output of cosmic rays would be expected to be comparable to that of the $\gamma$-rays and it appeared that Cygnus X-3 alone could account for all of the cosmic ray production in the Galaxy above $1 \mathrm{PeV}$ (Hillas 1984). There are, however, theoretical reasons to expect beaming in UHE binary sources (see, e.g., Hillas \& Johnson 1991) and this would reduce the source luminosity and cosmic-ray production.

Subsequent observations were made by several groups operating in the northern hemisphere during the 1980s. Many groups reported positive detections (see, e.g., Watson 1985 and Protheroe 1987 for reviews), and although none of these was of extremely high statistical significance there was quite good consistency in the reported orbital phase of emission which appeared to change with time but was always between 0.2 and 0.3 or between 0.5 and 0.7 . Figure 1 shows the orbital phase of $\gamma$-ray emission for detections claimed before 1987 August. It was largely these early results on Cygnus X-3 and other sources that stimulated the building of new air shower arrays designed specifically for $\gamma$-ray astronomy. Between the mid-1970s and mid-1980s the reported long-term average flux appeared to decrease steadily by at least a factor of 100 (Bhat, Sapru, \& Razdan 1986) as illustrated in Figure 2. Although this is extremely frustrating, it should perhaps have come as no surprise that the new air shower arrays did not detect steady emission from Cygnus X-3, and since 1986 there have been no statistically significant detections of steady emission at PeV energies of this source ( see Alexandreas et al. 1991 and Ciampa et al. 1992 for typical upper limits). The lowest upper limits from contemporary measurements are from Tibet AS- $\gamma$ (Amenomori et al. 1992) and CASA MIA (Borione et al. 1992), and they have been added to Figure 2.

\subsection{Burst Activity and Emission Associated with Radio Flaring}

Cygnus $\mathrm{X}-3$ is an extremely variable source at all wavelengths at which it has been studied. For example, the radio flux changes on time scales of a few days from less than $0.1 \mathrm{Jy}$ to greater than $10 \mathrm{Jy}$. UHE $\gamma$-ray emission has been detected at times of strong radio flaring during the 1972 September, 1980 October, 1985 October, and other flares (see, e.g., Protheroe 1987 for a discussion ). The 1985 October flare is particularly interesting because the Haverah Park, Baksan, and Akeno 1 $\mathrm{km}^{2}$ arrays detected fluxes apparently associated with the burst, as well as the Gulmarg, Durham, and Haleakala tele-

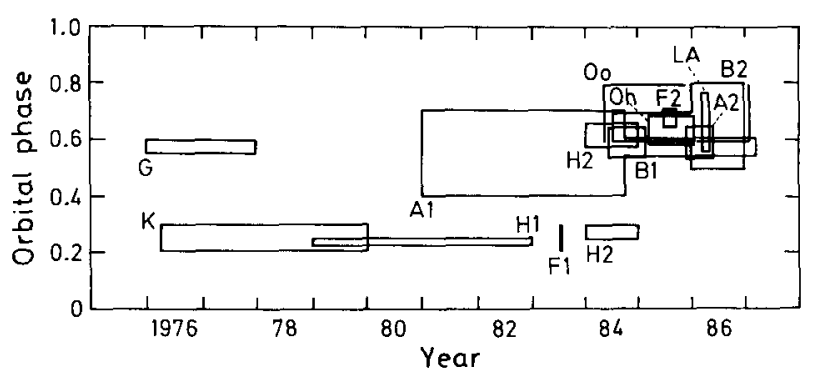

FiG. 1.-Orbital phase of $\gamma$-ray emission from Cygnus X-3 at energies above 0.1 PeV (from Protheroe 1987). See Nagle, Gaisser, \& Protheroe (1988) for key to data. 


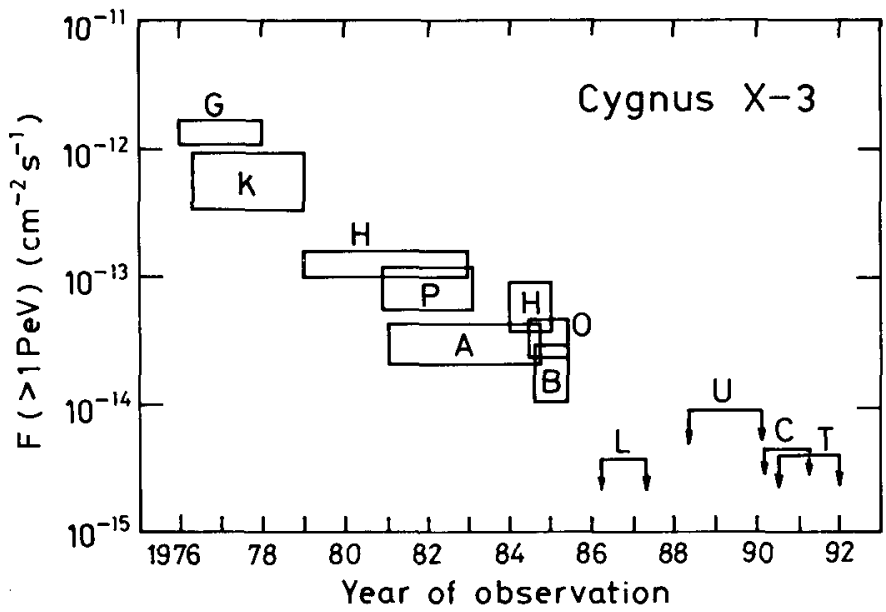

Fig. 2.-Fluxes from Cygnus X-3 above $1 \mathrm{PeV}$ corrected for absorption on the microwave background radiation plotted against year of observation (adapted from Bhat, Sapru, \& Razdan 1986). Key to data: G, Gulmarg; K, Kiel; H, Haverah Park; P, Plateu Rosa; A, Akeno; O, Ooty; B, Baksan; L, Los Alamos (Dingus et al. 1988); U, Utah-Michigan (Ciampa et al. 1992); C, CASA MIA (Borione et al. 1992); and T, Tibet AS- $\gamma$ (Amenomori et al. 1992); see Bhat, Sapru, \& Razdan 1986 for references for pre-1986 data. Upper limits are based on muon-poor shower data where available and have been adjusted to $1 \mathrm{PeV}$ assuming an $E^{-1}$ integral spectrum. A correction for absorption in the microwave background has been made using Fig. 8 of Nagle, Gaisser, \& Protheroe (1988).

scopes operating at $\mathrm{TeV}$ energies. In fairness, one should point out that the Plateau Rosa array, the Fly's Eye, and the Whipple telescope did not detect any emission, although this could be due a burst-like nature of the emission. This scenario is consistent with the Durham observations in which they only reported emission during two of the four nights they observed the source.

As well as at times of radio flaring, Cygnus X-3 has apparently had episodes of enhanced emission lasting several days or weeks. One such episode occurred during 1986. The CYGNUS array saw a DC excess in muon-poor showers from the direction of Cygnus X-3 during the 45 day interval starting on April 17 (Dingus et al. 1988). The events were found to be modulated with the $4.8 \mathrm{hr}$ period. Excesses were also seen during this interval at the Baksan (Alexeenko et al. 1987), Ooty (Tonwar et al. 1992), and Akeno (Teshima et al. 1990) arrays. The Ooty group examined their data to search for burst activity on shorter time scales by looking at the weekly occurrence of two events within 15 minutes of each other. They found that during the week of April 5 this number of "doubles" increased from an average of $\sim 0.6$ to 5 per week. Even taking into account the expected increase in the doubles rate because of the increase in the singles rate, they claim very strong evidence for burst-type activity on time scales of 15 minutes.

Perhaps even more striking is the period of enhanced emission in mid-June of 1985 . The Fly's Eye group reported a significant excess above $2.5 \times 10^{14} \mathrm{eV}$ on the night of June 17 (Baltrusaitis et al. 1987). Surprisingly, the emission occurred near orbital phase 0 . Two days later, the Ooty array observed six showers from the direction of Cygnus X-3 during a 40 minute interval (Tonwar et al. 1992). This was way above their average rate which was about one per day, and the orbital phase of emission was between 0.0 and 0.15 .

\subsection{Observations of Neutral Particles above $0.5 \mathrm{EeV}$}

There is strong evidence of emission of neutral particles from Cygnus X-3 at EeV $\left(10^{18} \mathrm{eV}\right)$ energies from at least two experiments. The Fly's Eye group analyzed their data taken between 1981 and 1988 and constructed a map of statistical significance of DC emission, as a function of direction, in showers of energy above $0.5 \mathrm{EeV}$ (Cassiday et al. 1989). They found that of all directions in the sky accessible to the latitude of the Fly's Eye, the direction of Cygnus X-3 had the highest significance with a $4 \sigma$ excess. At $1 \mathrm{EeV}$ the decay length of neutrons is $\sim 9 \mathrm{kpc}$, and so it is possible that the observed neutral particles are neutrons produced in interactions of protons or nuclei inside the source rather than $\gamma$-rays (Jones 1990; Capdevielle \& Gabinski 1991 ).

The Akeno group analyzed data from their $20 \mathrm{~km}^{2}$ array for the period from 1984 December to 1989 July, a period which overlaps that of the Fly's Eye observations (Teshima et al. 1990). The energy range, above $0.5 \mathrm{EeV}$, was the same as for Fly's Eye, and they too saw the largest DC excess $(3.5 \sigma)$ from a direction consistent with Cygnus X-3. During part of this time the Akeno group also operated a $1 \mathrm{~km}^{2}$ array with an energy threshold of $\sim 10 \mathrm{PeV}$, and they searched for correlated increases in the event rate of the two arrays for showers from the direction of Cygnus X-3. They found two such increases. The first of these, in 1986, coincided with the period of enhanced emission lasting $\sim 40$ days identified by the CYGNUS, Baksan, and Ooty arrays discussed in $\S 2.2$. The second correlated increase had a similar duration and occurred in March-April of 1989.

Data taken with the Akeno Giant Air Shower Array (AGASA), which has an area of $100 \mathrm{~km}^{2}$, between 1990 February and 1991 July also show the DC excess $(4 \sigma)$ from the same direction (Hayashida et al. 1991). In this case, all of the excess events are consistent with emission during a 50 day interval following the giant radio flare on 1991 January 20-21, and the energy spectrum of these excess showers is harder than the cosmic-ray background events. Interestingly, on January 20 and 23 a significant excess of muons from within $2^{\circ}$ of Cygnus $\mathrm{X}-3$ was seen in the Soudan 2 underground nucleon decay detector (Thomson et al. 1991), and the HEGRA array operating at PeV energies saw a $3.8 \sigma$ DC excess from Cygnus X-3 on January 20 although they claim no detection of the source (Merck et al. 1991). There is also an excess in data taken with CASA MIA on that day, although the excess $(\sim 1.7 \sigma)$ is not significant. Unfortunately, the hoped-for excess following the radio flare on 1991 July 26 did not materialize and as of 1993 February the cumulative DC excess in the direction of Cygnus $\mathrm{X}-3$ from AGASA was $1.5 \sigma(\mathrm{M}$. Teshima, personal communication).

Data from the Yakutsk array taken between 1974 and 1986, a time interval overlapping the Fly's Eye and Akeno observations, do contain evidence of a narrow angle anisotropy from the direction of Cygnus X-3 (Glushkov et al. 1990), although the authors suggest the excess may be due to streaming of cosmic rays along the "spiral arm in" direction (almost coincident with the direction of Cygnus X-3) rather than neutral particles. However, one would expect a simple dipole anisotropy rather than a narrow angle one for such a scenario. The evidence is present for a DC excess only in showers of energies 
0.2-0.3 EeV during 1984 to 1986 . Binning the sky in right ascension and declination, the only direction to have a DC excess greater than $3 \sigma$ is that of Cygnus X-3. Treating this as a point source observation, and taking account of the number of trials (energy ranges and time periods selected), I would estimate the chance probability to be $\sim 10^{-2}$. So, three separate experiments (Fly's Eye, Akeno, and Yakutsk) operating at the same time apparently saw much the same thing, the largest DC excess in the EeV energy range coming from the direction of Cygnus X-3. In addition, AGASA later saw a significant excess, apparently correlated with a radio flare in 1991 January. It is worrisome, however, that data from the Haverah Park array which was operational at the time of the Fly's Eye, Akeno, and Yakutsk observations showed no evidence for DC emission in this energy range from Cygnus X-3 (Lawrence, Prosser, \& Watson 1989).

\subsection{Evidence for $12.6 \mathrm{~ms}$ Pulse Period}

The Durham group found evidence for a pulse period of $12.5908 \mathrm{~ms}$ in their Cherenkov data taken in 1981 at an orbital phase of 0.6 , close to X-ray maximum (Chadwick et al. 1985). Subsequent observations made in 1982, 1983, 1985, and 1988 showed strong periodicity at essentially the same period-a reasonable period derivative of $\dot{P}=1.9 \times 10^{-14}$ gave an excellent fit to the periods obtained over this 5 year interval (Brazier et al. 1990). During their 1983 September 12 observations, the count rate increased by more than $10 \%$ and the combined chance probability of that night's observation and that on October 2 was $\sim 10^{-6}$.

In 1989 the Adelaide group, using their optical Cherenkov telescope at Woomera in Australia, observed this "Northern" source (Gregory et al. 1990). Because of the large zenith angles of observation, the energy threshold of the telescope increased from its usual $0.5 \mathrm{TeV}$ to $\sim 100 \mathrm{TeV}$, and the area of the Cherenkov light pool increased dramatically because for the large zenith angles the showers reach maximum development at a much larger distance from the telescope than in vertical showers (Sommers \& Elbert 1987). The observations were made during the night of July 27, 6 days after the start of a giant radio flare, and during the following 2 months. On July 27 the count rate increased by $\sim 40 \%$ above background for $\sim 15$ minutes. The data taken on the nights of August 26-28 and September 1-2 showed strong periodicity at $12.596 \mathrm{~ms}$ with a chance probability of $10^{-3}$ (if the DC excess in the data taken on July 27 were used also in the analysis, the probability would decrease dramatically). The period found is consistent with the earlier Durham observations, given that the pulse period may have had a spin-up or spin-down episode of the type observed in other X-ray binaries since the 1988 Durham observations. The Durham group also observed Cygnus X-3 in September (Bowden et al. 1992) and on September 1 and 7 there was evidence of emission at the $12.59 \mathrm{~ms}$ period with chance probabilities of $10^{-3}$ and $3 \times 10^{-6}$.

Several other groups have attempted to observe the $12 \mathrm{~ms}$ period in Cygnus X-3 at TeV energies without success (e.g., O'Flaherty et al. 1992). Part of the reason for this may be in the different types of telescope used-the Durham and Adelaide systems at that time were not imaging systems and had three mirrors on the same mount with a triple coincidence trigger. This meant that the event rates were high and included events with too few photons to give images which would satisfy the requirements of a $\gamma$-ray image in the Whipple telescope. The higher event rates are helpful for pulsar studies. The most likely reason is, however, the highly variable nature of the emission as already noted. The Durham group made 168 observations which cover the passage of X-ray maximum and on five or six occasions they observed a reasonably good signal for a few minutes. In contrast, observations by the Whipple group (O'Flaherty et al. 1992) include 24 scans which cover this orbital phase, and they saw no count rate increase or evidence of periodicity. Based on the Durham results, if the source is "on" during X-ray maxima only $\sim 4 \%$ of the time, then in 24 observations one would have an expectation of seeing less than one Durham-type outburst (A. M. Hillas, personal communication ). All other groups have observed the source on fewer occasions than the Durham group.

\section{CRAB NEBULA}

During the last few years the Whipple collaboration has demonstrated that at $\mathrm{TeV}$ energies the imaging technique can be used to reject a large fraction of the cosmic-ray background when using the Cherenkov technique. Their $20 \sigma$ detection of the Crab Nebula (Vacanti et al. 1991) is universally accepted in the $\gamma$-ray astronomy community and has been confirmed by observations made in France by the ASGAT group (Goret et al. 1993) and at somewhat higher energies by the THEMISTOCLE group (Baillon et al. 1993). A number of models of the Crab Nebula/Pulsar have been proposed which predict VHE $\gamma$-ray emission (e.g., Cheng et al. 1990; Kwock, Cheng, \& Lau 1991). One of the most promising is the pulsar wind shock acceleration model of De Jager \& Harding (1992) in which electrons inverse-Compton scatter lower energy radiation from synchrotron emission in a realistic magnetic field model. Their model gives an excellent fit to the $C O S-B$ data and the VHE observations, and predicts observable fluxes at $100 \mathrm{TeV}$ energies. The TIBET AS- $\gamma$ (Amenomori et al. 1992) and CASA MIA (Borione et al. 1992) arrays have placed upper limits on the steady long-term emission which are not inconsistent with the model predictions. Figure 3 shows a summary of the measured fluxes compared with the model of De Jager \& Harding (1992). In fact, CASA MIA sees a $1.8 \sigma$ DC excess in muon-poor showers with size above $2.2 \times 10^{4}$ which, if taken as a flux, would be on a direct power-law extrapolation of the Whipple and THEMISTOCLE observations. It is expected that with a further year's observing a significant excess would be detected by CASA MIA if the flux were indeed this high.

Since the Whipple observations were reported, much has been made of the apparent steady nature of the emission and the possibility that the Crab may be regarded as a "standard candle" in $\gamma$-ray astronomy. There are, however, strong indications that this may not be the case at UHE energies. On 1989 February 23 three separate groups detected excess events from the direction of the Crab above $100 \mathrm{TeV}$. Because the sensitivity of an air shower array is strongly dependent on zenith angle, a typical array at midlatitudes can only "see" a particular source for $\sim 6 \mathrm{hr}$ each day. (Note, however, this does not apply to polar arrays which observe sources 24 hours a day at constant zenith angle.) When the Crab came into view of the Kolar Gold Fields array in India at $\sim$ 13:00 UT there was an immediate increase in event rate to more than twice that expected 


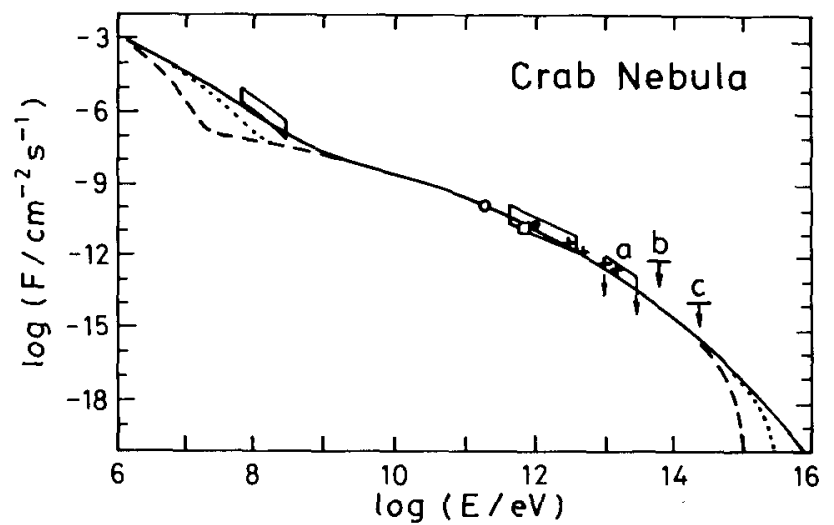

FIG. 3.-Crab Nebula flux expected in the model of De Jager \& Harding (1992) compared with recent observations and upper limits (adapted from De Jager \& Harding 1992). The different curves are for different maximum energies in the model: dashed line, $10^{15}$; dotted line, $3 \times 10^{15}$; solid line, $10^{16} \mathrm{eV}$. Key to data: open rectangle at $100 \mathrm{MeV}, \operatorname{COS}-B$ (Clear et al. 1987); open circle, Gamma* (Akerlof et al. 1990); open square, Whipple (Weekes et al. 1989); filled circle, ASGAT (Goret et al. 1993); open rectangle at $1 \mathrm{TeV}$, Whipple (Vacanti et al. 1991); plus sign, THEMISTOCLE (Baillon et al. 1993); "a," Tibet AS- $\gamma$ (Amenomori et al. 1992); "b," CYGNUS (Alexandreas et al. 1991); "c," Utah-Michigan (Ciampa et al. 1992). The CASA MIA upper limit (Borione et al. 1992) is presently almost identical to the Utah-Michigan limit.

from cosmic rays alone (Acharya et al. 1990, 1991). Unfortunately, the other important array in India, at Ooty, was not fully operational at the time of the burst (S. C. Tonwar, personal communication ). About $2 \mathrm{hr}$ later the source was visible from the Baksan (Alexeenko et al. 1992) and Tien Shan (referred to by Alexeenko et al. 1992) arrays in Russia which both saw a similar excesses, and the story was repeated $2 \mathrm{hr}$ after that when the EAS Top array in Italy could see the source (Aglietta et al. 1991). The event rates and expected backgrounds are shown in Figure 4 for the Kolar Gold Fields, Baksan, and EAS Top arrays. By the time the Crab became visible at the HEGRA array in the Canary Islands, the UHE $\gamma$-ray emission had apparently ceased, and no evidence for UHE emission was found in the data of the SPICA array in Japan for emission earlier than that detected in India. This indicates that the UHE $\gamma$-ray burst from the Crab had a duration less than $12 \mathrm{hr}$.

The excesses in standard deviations ( $\mathrm{Li} \& \mathrm{Ma} 1983$ ) seen by the Kolar Gold Fields, Tien Shan, Baksan, and EAS Top arrays ( 2 independent data sets) are 3.4, 2.6, 3.1, 2.1, and 1.2 $\sigma$. Combining these data (Eadie et al. 1971) gives an overall probability of $1.7 \times 10^{-5}$ of these observations occurring by chance (Alexeenko et al. 1992). There is also some evidence of the emission being pulsed at the Crab pulsar period in the Baksan and Kolar Gold Fields data. So, it would appear that even this "standard candle" may show the strong time-variability which seems to characterize UHE $\gamma$-ray sources like Cygnus X-3. Indeed, earlier observations by the Ooty group indicate that the Crab Nebula/Pulsar has been a sporadic UHE $\gamma$-ray source (Gupta et al. 1991).

\section{CONCLUSIONS}

It is my opinion that Cygnus X-3 was a source of UHE $\gamma$-rays from the mid 1970s to the mid 1980s, and that the source is now in a state of reduced activity at UHE. The fluxes reported over this period showed a steady decline, with typical fluxes in 1985 being a factor of $\sim 100$ lower than those observed around 1975. There is evidence that other binary X-ray sources have shown variations in activity with active and inactive cycles lasting many years. One example is Hercules X-1 for which optical observations go back over $100 \mathrm{yr}$ (Jones, Forman, \& Liller 1973; Hudec \& Wenzel 1986, and references therein). In this source, a substantial fraction of the optical emission comes from the accretion disk and the light curve is at a minimum when the disk is partly occulted by the star $(\phi=$ 0 ). When the source is inactive, and the accretion disk is not present, the photographic magnitude increases by $\sim 2$ and the light curve changes to have two minima (instead of one) at $\phi=$ 0 and $\phi=0.5$ corresponding to the Roche lobe having the smallest projected area. Since 1890 Hercules X-1 has had four active states, the present one starting in 1957. This shows that it is certainly not unreasonable for $X$-ray binaries such as Cygnus X-3 to have turned off their UHE emission temporarily.

The nature of the UHE emission from Cygnus X-3 has been sporadic, with variability on time scales from several minutes to years. Emission sometimes occurred around the time of, or soon after, a giant radio flare. The UHE $\gamma$-ray flux was found to be modulated by the $4.8 \mathrm{hr}$ orbital period in many observations up to the mid 1980s. The emission occurred mainly at orbital phase $0.2-0.3$ or $0.5-0.7$. There is evidence for pulsa-
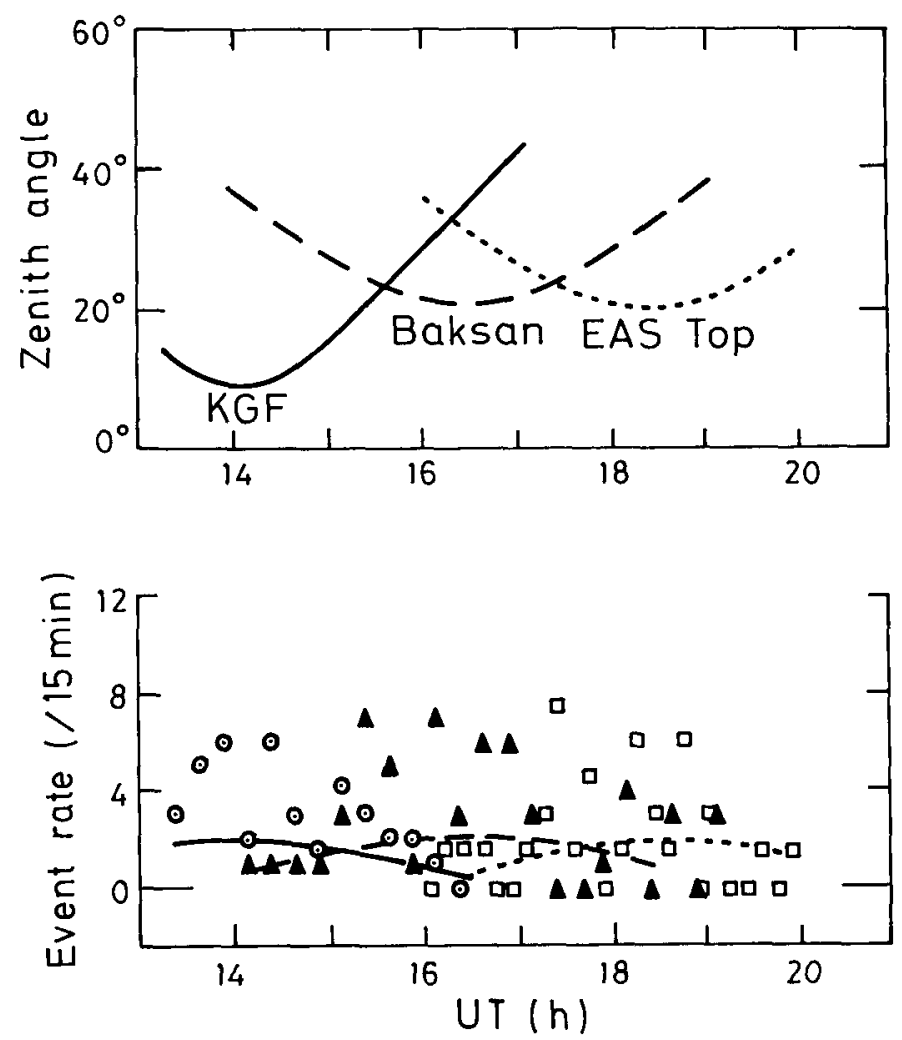

Fig. 4.-The UHE $\gamma$-ray burst from the Crab Nebula on 1989 February 23: (a) zenith angle of the source as seen at the Kolar Gold Fields, Baksan, and EAS Top arrays; $(b)$ count rate from the direction of the Crab seen in the three arrays compared with the background (adapted from Acharya et al. 1991). Key to data: open circles: Kolar Gold Fields; filled triangles: Baksan; open squares: EAS Top. 
tion at $12.6 \mathrm{~ms}$ at $\mathrm{TeV}$ to $\sim 100 \mathrm{TeV}$ energies in optical Cherenkov data. Emission of neutral particles (presumably either $\gamma$-rays or neutrons) at energies around $0.5 \mathrm{EeV}$ was observed in the late 1980s in at least two UHE cosmic-ray observatories.

As well as the very convincing $\mathrm{TeV}$ observations of apparently steady emission from the Crab Nebula, there is also strong evidence that the Crab is a source of sporadic UHE emission. In 1989 three independent experiments monitored a burst at $\mathrm{PeV}$ energies over a $\sim 12 \mathrm{hr}$ interval.

In conclusion, I do not believe that the Crab Nebula is the only Galactic $\gamma$-ray source observed at or above $\mathrm{TeV}$ energies, or that its flux is constant. The weight of evidence of UHE and VHE emission from Cygnus X-3 I find fairly convincing, and the data show that this source was highly variable at UHE. Recent UHE $\gamma$-ray observations of a burst on 1989 February 23 indicate that the Crab Nebula/Pulsar is also highly variable.

I thank E. Chupp for the opportunity to attend IAU Colloquium 142. I am grateful to R. Clay, B. Dawson, P. Edwards, and $\mathrm{G}$. Thornton for helpful discussions and for reading the manuscript, and to $P$. Johnson for bringing to my attention the optical observations of Hercules X-1 spanning over a century.

\section{REFERENCES}

Acharya, B. S., Rao, M. V. S., Sivaprasad, K., Sreekantan, B. V., \& Vishwanath, P. R. 1990, Nature, 347, 364

1991, High-Energy Gamma-Ray Astronomy, ed. J. Matthews

(New York: AIP), 137

Aglietta, M., et al. 1991, Europhys. Lett., 15(1), 81

Akerlof, C. W., et al. Nucl. Phys. B (Proc. Suppl.), 14A, 237

Alexandreas, D. E., et al. 1991, ApJ, 383, L53

Alexeenko, V. V., et al. 1987, in Proc. 20th Internat. Cosmic-Ray Conf. (Moscow), 1, 219 1992, J. Phys. G: Nucl. Part Phys., 18, L83

Amenomori, M., et al. 1992, Phys. Rev. Lett., 69, 2468

Baillon, P., et al. 1993, in Proc. 21st Internat. Conf. on High-Energy Physics (Dallas), in press

Baltrusaitis, R. M., et al. 1987, ApJ, 323, 685

Bhat, C. L., Sapru, M. L., \& Razdan, H., 1986, ApJ, 306, 587

Borione, A., et al. 1992, in Proc. APS Division of Particles and Fields Conf. (Fermilab), in press

Bowden, C. C. G., et al. 1992, J. Phys. G: Nucl. Part. Phys., 18, 413

Brazier, K. T. S., et al. 1990, ApJ, 350, 745

Capdevielle, J. N., \& Gabinski, P. 1991, in Proc. 22d Internat. Cosmic-Ray Conf. (Dublin), 1, 576

Cassiday, G. L., et al. 1989, Phys. Rev. Lett., 62, 383

Chadwick, P. M., et al. 1985, Nature, 318, 642

Cheng, K. S., Cheung, T., Lau, M. M., Yu, K. N., \& Kwock, P. W. 1990, J. Phys. G: Nucl. Part. Phys., 16, 1115

Ciampa, D. P., et al. 1992, Phys. Rev. D, 46, 3248

Clear, J., et al. 1987, A\&A, 174, 85

De Jager, O. C., \& Harding, A. K. 1992, ApJ, 396, 161

Dingus, B. L., et al. 1988, Phys. Rev. Lett., 60, 1785

Eadie, W. T., et al. 1971, Statistical Methods in Experimental Physics (Amsterdam: North Holland)

Fegan, D. J. 1990, in Proc. 21 st Internat. Cosmic Ray Conf. (Adelaide), 11,23

Gaisser, T. K., Stanev, T., \& Halzen, F. 1991, Proc. 22d Internat. CosmicRay Conf. (Dublin), 1, 564

Glushkov, A. V., et al. 1990, in Proc. 21 st Internat. Cosmic Ray Conf. (Adelaide), 2, 64

Goret, P., et al. 1993, A\&A, in press

Gregory, A. G., Patterson, J. R., Roberts, M. D., Smith, N. I., \& Thornton, G. J. A\&A, 237, L5
Gupta, S. K., Rajeev, M. R., Sreekantan, B. V., Srivatsan, R., \& Tonwar, S. C. 1991, A\&A, 245, 141

Hayashida, N., et al. 199l, in Proc. 22d Internat. Cosmic-Ray Conf. (Dublin), 1,309

Hillas, A. M. 1984, Nature, 312,50

Hillas, A. M., \& Johnson, P. A. 1991, in Proc. 22d Internat. Cosmic Ray Conf. (Dublin), 2, 452

Hudec, R., \& Wenzel, W. 1986, A\&A, 158, 396

Ip, W.-H., \& Axford, W. I. 1992, Particle Acceleration in Cosmic Plasmas, ed. G. P. Zank \& T. K. Gaisser (New York: AIP), 400

Jones, C. A., Forman, W., \& Liller, W. 1973, ApJ, 182, L109

Jones, L. W. 1990, in Proc. 21 st Internat. Cosmic Ray Conf. (Adelaide), 2

Kwock, P. W., Cheng, K. S., \& Lau, M. M. 1991, ApJ, 379, 653

Lawrence, M. A., Prosser, D. C., \& Watson, A. A. 1989, Phys. Rev. Lett., 63,1121

Li, T. P., \& Ma, Y. Q. 1983, ApJ, 272, 317

Merck, M., et al. 1991, in Proc. 22d Internat. Cosmic Ray Conf. (Dublin), 1,261

Nagle, D. E., Gaisser, T. K., \& Protheroe, R. J. 1988, Ann. Rev. Nucl. Part. Sci., 38, 609

O'Flaherty, K. S., et al. 1992, ApJ, 396, 674

Ong, R. A. 1992, in Proc. 14th Rencontre de Blois-Particle Astrophysics, ed. J. Tran Thanh Van (Gif sur Yvette, Editions Frontières), in press Protheroe, R. J. 1986, MNRAS, 221, 769

- 1987, in Proc. 20th Internat. Cosmic-Ray Conf. (Moscow), 8, 21

Protheroe, R. J., \& Szabo, A. P. 1992, Phys. Rev. Lett., 69, 2885

Rao, M. V. S., \& Sreekantan, B. V. 1992, Current Sci., 62, 617

Samorski, M., \& Stamm, W. 1983, ApJ, 268, L.17

Sommers, P., \& Elbert, J. W. 1987, J. Phys. G: Nucl. Phys., 13, 553

Teshima, M., et al. 1990, Phys. Rev. Lett., 64, 1628

Thomson, M. A., et al. 1991, Phys. Lett., B, 269, 220

Tonwar, S. C., Gupta, S. K., Rajeev, M. R., Sreekantan, B. V., \& Srivatsan, R. 1992, ApJ, 390, 273

Vacanti, G., et al. 1991, ApJ, 377, 467

Watson, A. A. 1985, in Proc. 19th Internat. Cosmic Ray Conf. (La Jolla) 9,111

Weekes, T. C. 1988, Phys. Rep., 160, 2

- 1991, Space Sci. Rev., 59, 315

Weekes, T. C., et al. 1989, ApJ, 342, 379 